\title{
Article \\ Inhibitory Effect of a Glutamine Antagonist on Proliferation and Migration of VSMCs via Simultaneous Attenuation of Glycolysis and Oxidative Phosphorylation
}

\author{
Hyeon Young Park ${ }^{1,2,+}$, Mi-Jin Kim ${ }^{3,4,+}$, Seunghyeong Lee ${ }^{1,2}$, Jonghwa Jin ${ }^{3}$, Sungwoo Lee ${ }^{5}$, Jung-Guk Kim ${ }^{3}$, \\ Yeon-Kyung Choi ${ }^{3,4, *}$ and Keun-Gyu Park ${ }^{1,3,4, *(D)}$
}

Citation: Park, H.Y.; Kim, M.-J.; Lee, S.; Jin, J.; Lee, S.; Kim, J.-G.; Choi, Y.-K.; Park, K.-G. Inhibitory Effect of a Glutamine Antagonist on Proliferation and Migration of VSMCs via Simultaneous Attenuation of Glycolysis and Oxidative Phosphorylation. Int. J. Mol. Sci. 2021, 22, 5602. https://doi.org/ 10.3390/ijms22115602

Academic Editor: Ana Belen Garcia-Redondo

Received: 29 April 2021

Accepted: 21 May 2021

Published: 25 May 2021

Publisher's Note: MDPI stays neutral with regard to jurisdictional claims in published maps and institutional affiliations.

Copyright: (c) 2021 by the authors. Licensee MDPI, Basel, Switzerland. This article is an open access article distributed under the terms and conditions of the Creative Commons Attribution (CC BY) license (https:/ / creativecommons.org/licenses/by/ $4.0 /)$.
1 Department of Biomedical Science, Graduate School, Kyungpook National University, Daegu 41566, Korea; coolphyp@gmail.com (H.Y.P.); ngell92@gmail.com (S.L.)

2 BK21 FOUR KNU Convergence Educational Program of Biomedical Sciences for Creative Future Talents, School of Medicine, Kyungpook National University, Daegu 41566, Korea

3 Department of Internal Medicine, School of Medicine, Kyungpook National University, Kyungpook National University Hospital, Daegu 41944, Korea; kij200@nate.com (M.-J.K.); becauseofu77@gmail.com (J.J.); jugkim@knu.ac.kr (J.-G.K.)

4 Research Institute of Aging and Metabolism, Kyungpook National University, Daegu 41566, Korea

5 New Drug Development Center, Daegu Gyeongbuk Medical Innovation Foundation, Daegu 41061, Korea; swlee@dgmif.re.kr

* Correspondence: ykchoi@knu.ac.kr (Y.-K.C.); kpark@knu.ac.kr (K.-G.K.)

+ These authors contributed equally.

Abstract: Excessive proliferation and migration of vascular smooth muscle cells (VSMCs) contribute to the development of atherosclerosis and restenosis. Glycolysis and glutaminolysis are increased in rapidly proliferating VSMCs to support their increased energy requirements and biomass production. Thus, it is essential to develop new pharmacological tools that regulate metabolic reprogramming in VSMCs for treatment of atherosclerosis. The effects of 6-diazo-5-oxo-L-norleucine (DON), a glutamine antagonist, have been broadly investigated in highly proliferative cells; however, it is unclear whether DON inhibits proliferation of VSMCs and neointima formation. Here, we investigated the effects of DON on neointima formation in vivo as well as proliferation and migration of VSMCs in vitro. DON simultaneously inhibited FBS- or PDGF-stimulated glycolysis and glutaminolysis as well as mammalian target of rapamycin complex I activity in growth factor-stimulated VSMCs, and thereby suppressed their proliferation and migration. Furthermore, a DON-derived prodrug, named JHU-083, significantly attenuated carotid artery ligation-induced neointima formation in mice. Our results suggest that treatment with a glutamine antagonist is a promising approach to prevent progression of atherosclerosis and restenosis.

Keywords: glutamine antagonist; vascular smooth muscle cells; glycolysis; oxidative phosphorylation; mTORC1

\section{Introduction}

Vascular smooth muscle cells (VSMCs) are the major component of the vasculature and help to maintain vessel tone, the bloodstream, and blood pressure [1]. Fully differentiated VSMCs remain in a non-proliferative state under normal conditions, while VSMCs dedifferentiate and their proliferation rate increases upon vascular injury [2,3]. Phenotypic switching of VSMCs in response to various physiological and pathological factors has long been considered of fundamental importance for proliferation and migration of VSMCs and pathological intima formation, which leads to the development of various vascular diseases including atherosclerosis, transplant vasculopathy, and pulmonary hypertension [4,5]. Thus, better understanding of the underlying mechanism might lead to the identification of a new therapeutic target for neointimal hyperplasia. 
Under physiological conditions, VSMCs undergo metabolic reprogramming such as enhancement of aerobic glycolysis to support their increased energy requirements and biosynthesis of macromolecules during rapid proliferation and contractile switching [6-8]. Previous studies demonstrated that enhanced glycolysis is highly required during proliferation of VSMCs by showing that glycolytic enzyme expression, lactate production, and glucose utilization are increased in growth factor-stimulated VSMCs [9-12]. Rapidly proliferating VSMCs also have an increased demand for glutamine, which is the most abundant circulating nutrient and is needed to maintain the redox balance, amino acid production, nucleotide synthesis, and extracellular matrix production $[13,14]$. Increasing evidence shows that enhanced glycolysis and glutaminolysis contribute to proliferation, migration, and collagen synthesis of vascular cells $[15,16]$. Therefore, therapeutic strategies based on the blockade of aerobic glycolysis or glutamine metabolism may be effective for treatment of occlusive vascular diseases.

6-diazo-5-oxo-L-norleucine (DON) is an L-glutamine antagonist that was discovered in the 1950s as a natural product of Streptomyces bacteria [17]. DON robustly inhibits cell proliferation by blocking glutamine-dependent enzyme pathways including those involving glutaminase 1 [18]. Despite the efficacy of DON in preclinical and clinical studies, its substantial peripheral toxicity has hampered its approval as a chemotherapeutic agent $[18,19]$. On the other hand, ethyl 2-(2-amino-4-methylpentanamido)-DON (JHU$083)$ is a DON-derived prodrug $[20,21]$. This inert glutamine antagonist is activated via proteolytic cleavage by enriched enzymes such as cathepsin-L in vivo, leading to the release of active DON [20,22]. Thus, JUH-083 exhibits increased oral bioavailability and decreased toxicity [21]. Although the anti-proliferative effects of glutamine antagonists have been broadly investigated in many types of cancer $[20,23,24]$, their effects on proliferation of VSMCs and ability to halt restenosis remain to be elucidated.

In this study, we investigated whether the glutamine antagonist DON and the DONderived prodrug JHU-083 inhibit proliferation and migration of VSMCs and suppress neointima formation in a mouse carotid artery ligation model.

\section{Results}

2.1. DON Attenuates Fetal Bovine Serum (FBS)-and Platelet-Derived Growth Factor (PDGF)-Induced Upregulation of Mitochondrial Respiration in VSMCs

Given that glutamine contributes to TCA intermediates and DON targets mitochondria in cancer research [25], we first measured the oxygen consumption ratio (OCR) in DONtreated VSMCs stimulated with FBS or PDGF. Basal, maximal, and ATP-linked respiration was increased upon FBS or PDGF stimulation; however, DON significantly decreased all these respiratory parameters (Figure 1A-D).

\subsection{DON Attenuates FBS- and PDGF-Induced Upregulation of Mammalian Target of Rapamycin Complex I (mTORC1) Activity and Glycolysis in VSMCs}

Glutaminolysis regulates activation of mTORC1 [26]. Thus, we investigated whether DON regulates mTORC1 activity in cultured VSMCs by measuring phosphorylation of $70 \mathrm{kDa}$ ribosomal protein $\mathrm{S6}$ kinase (p70S6K) and eukaryotic initiation factor 4E-binding protein 1 (4E-BP1), which are downstream substrates of mTORC1. DON attenuated the FBSand PDGF-induced increases in p-p70S6K and p-4E-BP1 expression (Figure 2A,B). Given that $\mathrm{mTORC} 1$ increases glucose uptake and glycolysis by upregulating hypoxia-inducible factor- $1 \alpha(\mathrm{HIF}-1 \alpha)$ [27], we next investigated whether the DON-induced suppression of mTORC1 inhibits glycolysis in VSMCs. The levels of HIF- $1 \alpha$ and its downstream targets hexokinase 2 (HK2) and lactate dehydrogenase A (LDHA) were increased in FBS- and PDGF-stimulated VSMCs, and downregulated by DON (Figure 3A,B). Consistent with the downregulation of HIF- $1 \alpha, \mathrm{HK} 2$, and LDHA, DON suppressed glycolysis and the glycolytic capacity in FBS- and PDGF-simulated VSMCs, as determined by measuring the extracellular acidification rate (ECAR) (Figure 3C-F). Overall, these results indicate that blockade of glutamine using DON significantly inhibits mTORC1 activity and HIF- $1 \alpha$ expression, which in turn suppresses glycolysis in growth factor-stimulated VSMCs. 
A

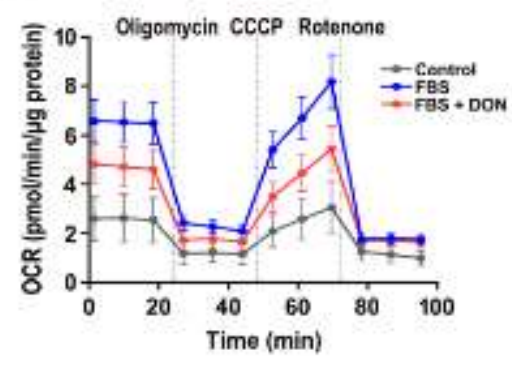

C

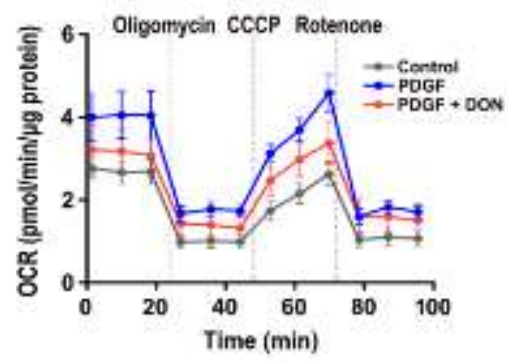

B
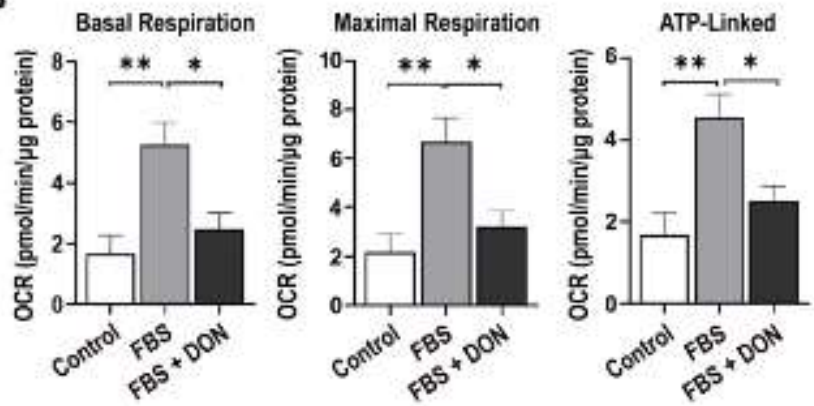

D
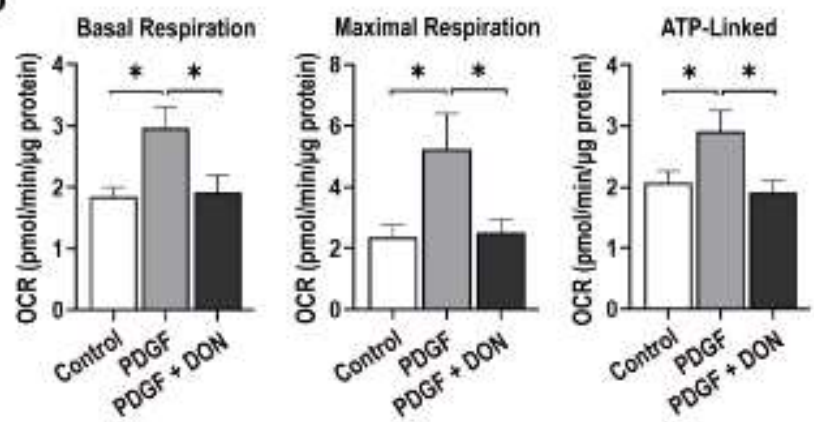

Figure 1. DON reduces mitochondrial respiration in growth factor-stimulated VSMCs. (A) and (C) OCR kinetic traces in FBS (A)- and PDGF (C)-stimulated VSMCs treated with or without DON. (B) and (D) Rates of basal, maximal, and ATP-linked respiration in FBS (B)- and PDGF(D)-stimulated VSMCs treated with or without DON. Data are expressed as the mean $\pm \operatorname{SEM}\left(n=3\right.$ technical replicates). ${ }^{*} p<0.05$ and ${ }^{* *} p<0.01$.

A
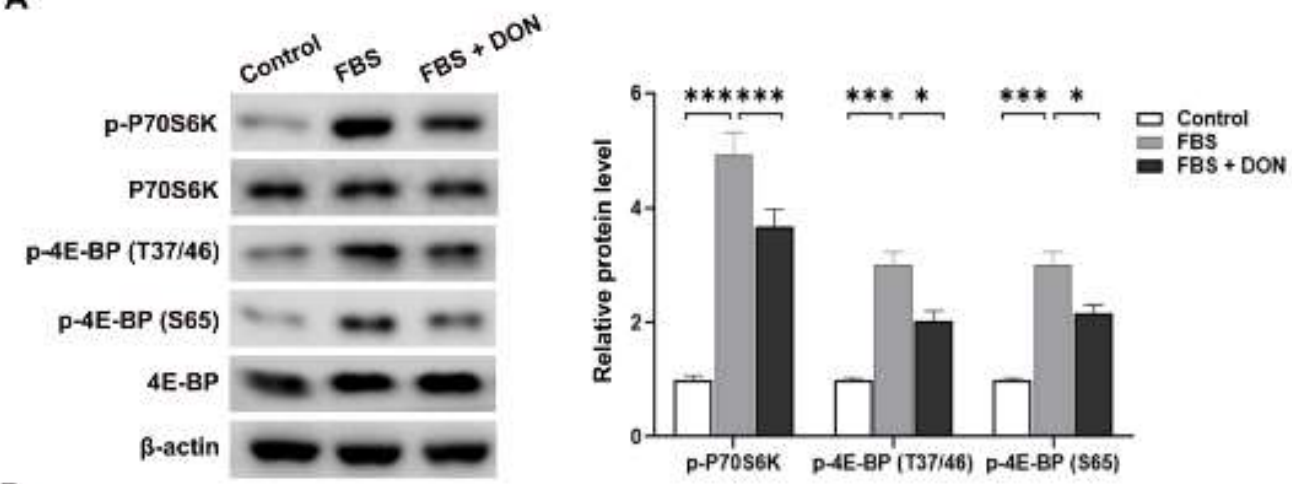

B
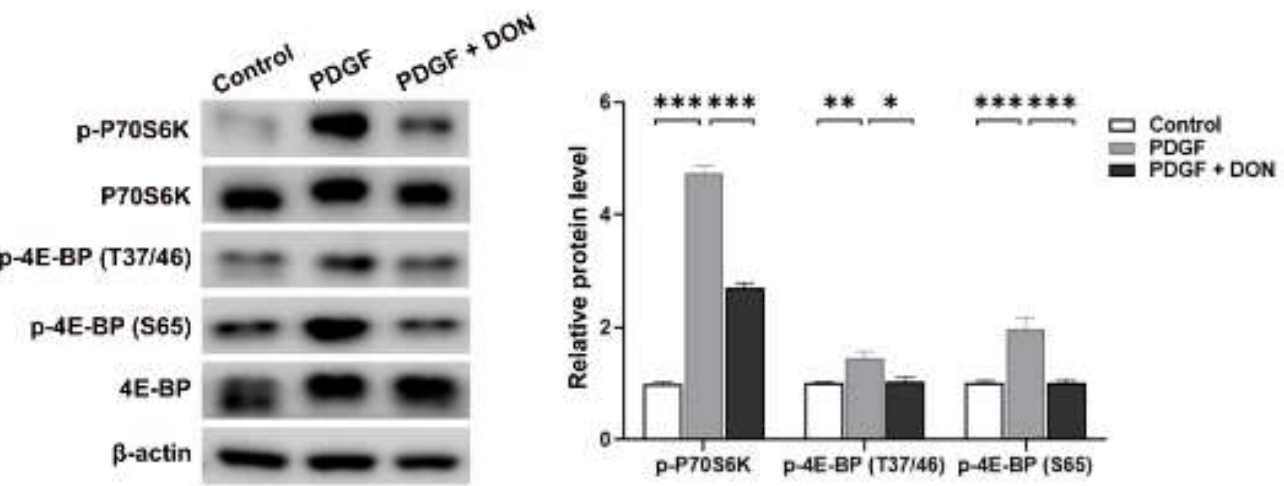

Figure 2. DON attenuates mTORC1 activity in growth factor-stimulated VSMCs. (A) and (B) Representative western blots showing the effects of DON on the levels of p-p70S6K, p-70S6K, 4E-BP, p-4E-BP (Thr37/46), and p-4E-BP (Ser65) in VSMCs stimulated with FBS (A) and PDGF (B). Data are expressed as the mean $\pm \operatorname{SEM}\left(n=3\right.$ technical replicates). ${ }^{*} p<0.05$, ${ }^{* *} p<0.01$, and ${ }^{* * *} p<0.001$. 
A
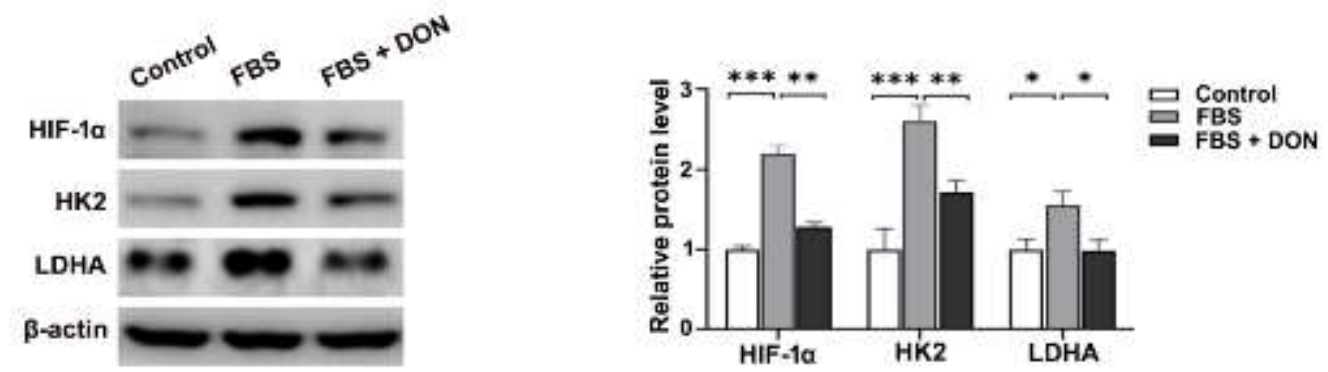

B
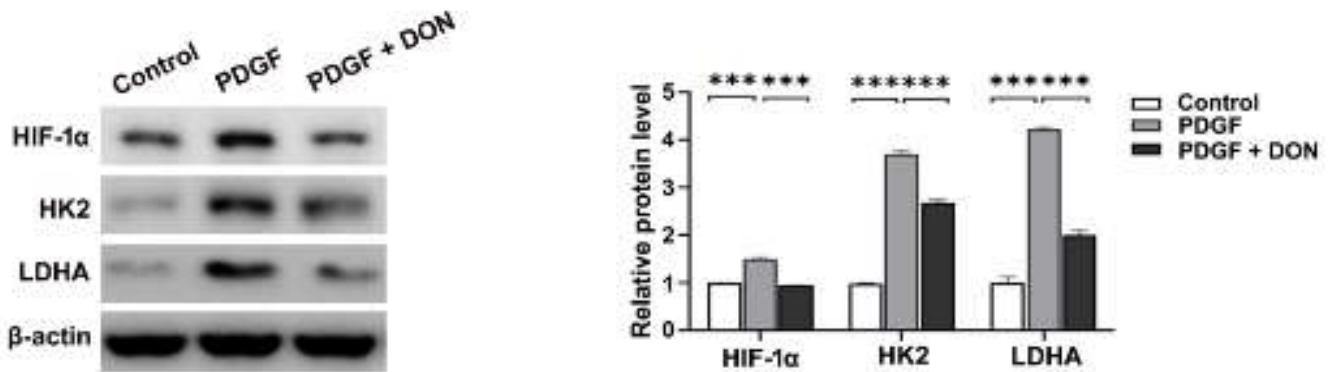

C

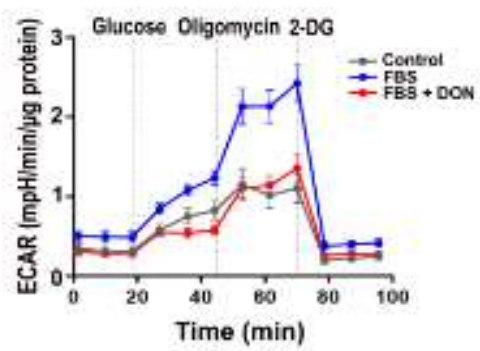

E

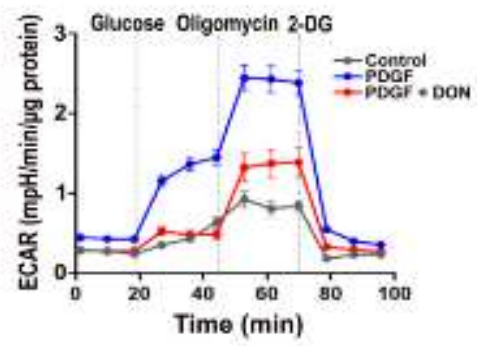

D

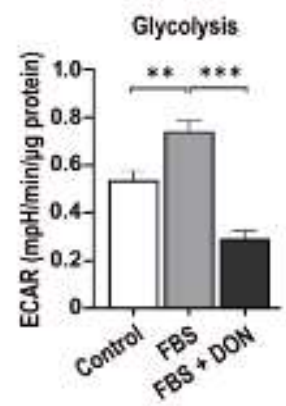

F

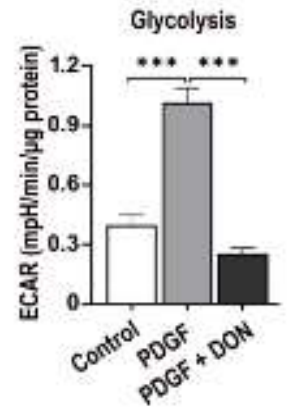

Glycolytic Capacity

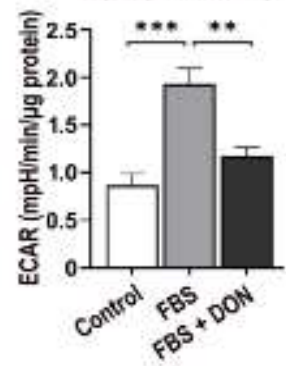

Glycolytic Capacity

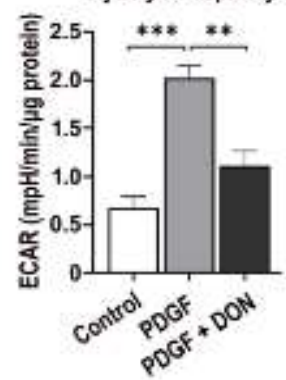

Figure 3. DON suppresses glycolysis in growth factor-stimulated VSMCs. (A) and (B) Representative western blots showing the effects of DON on the levels of HIF-1 $\alpha$, HK2, and LDHA in VSMCs stimulated with FBS (A) and PDGF (B). Data in bar graphs are mean \pm SEM of three independent measurements. (C)-(F) The ECAR in FBS- and PDGF-stimulated VSMCs. ECAR kinetic traces and the glycolytic rate and glycolytic capacity of FBS (C) and (D)- and PDGF (E) and (F)- stimulated VSMCs treated with or without DON. Data are expressed as the mean \pm SEM ( $n=3$ technical replicates). ${ }^{*} p<0.05$, ** $p<0.01$, and ${ }^{* * *} p<0.001$.

2.3. DON Delays Cell Cycle Progression and Inhibits Proliferation and Migration of FBS- and PDGF-Stimulated VSMCS

mTORC1 regulates cell cycle progression [28,29]; therefore, we investigated whether DON affects cell cycle progression of FBS- and PDGF-stimulated VSMCs. Western blot analysis revealed that the level of cyclin D1, a key regulator of VSMC proliferation, increased in response to FBS and PDGF, and this effect was reversed by DON (Figure 4A,B). Furthermore, flow cytometric analysis of the cell cycle showed that DON attenuated FBSand PDGF-stimulated progression from G1 to S phase (Figure 4C,D). 
A

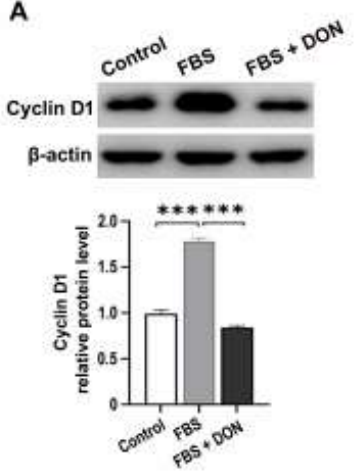

C
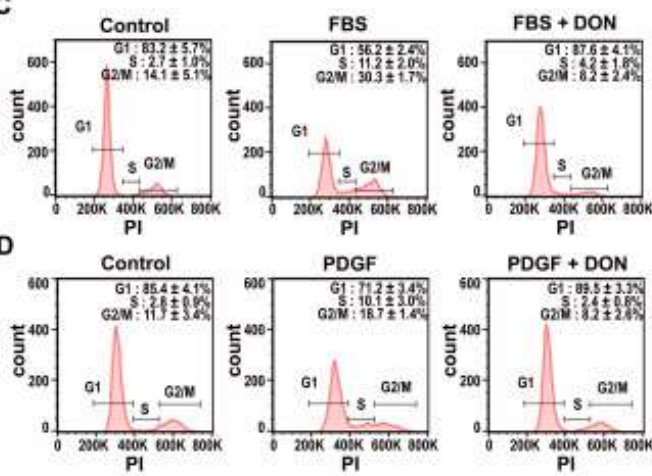

B

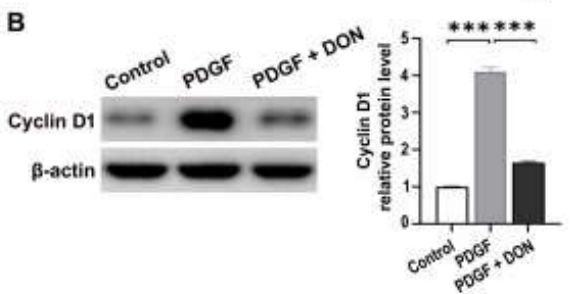

E
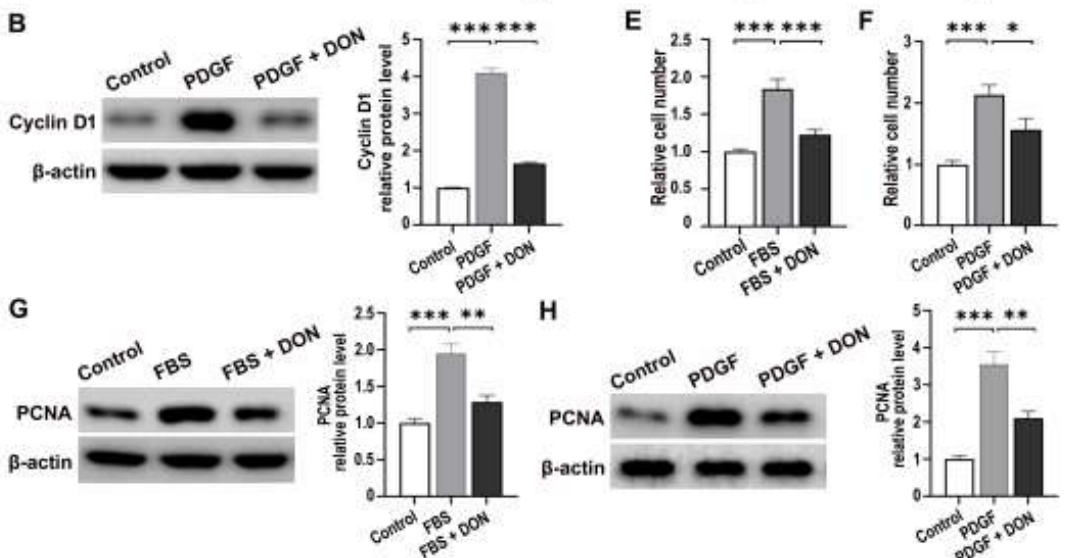

H
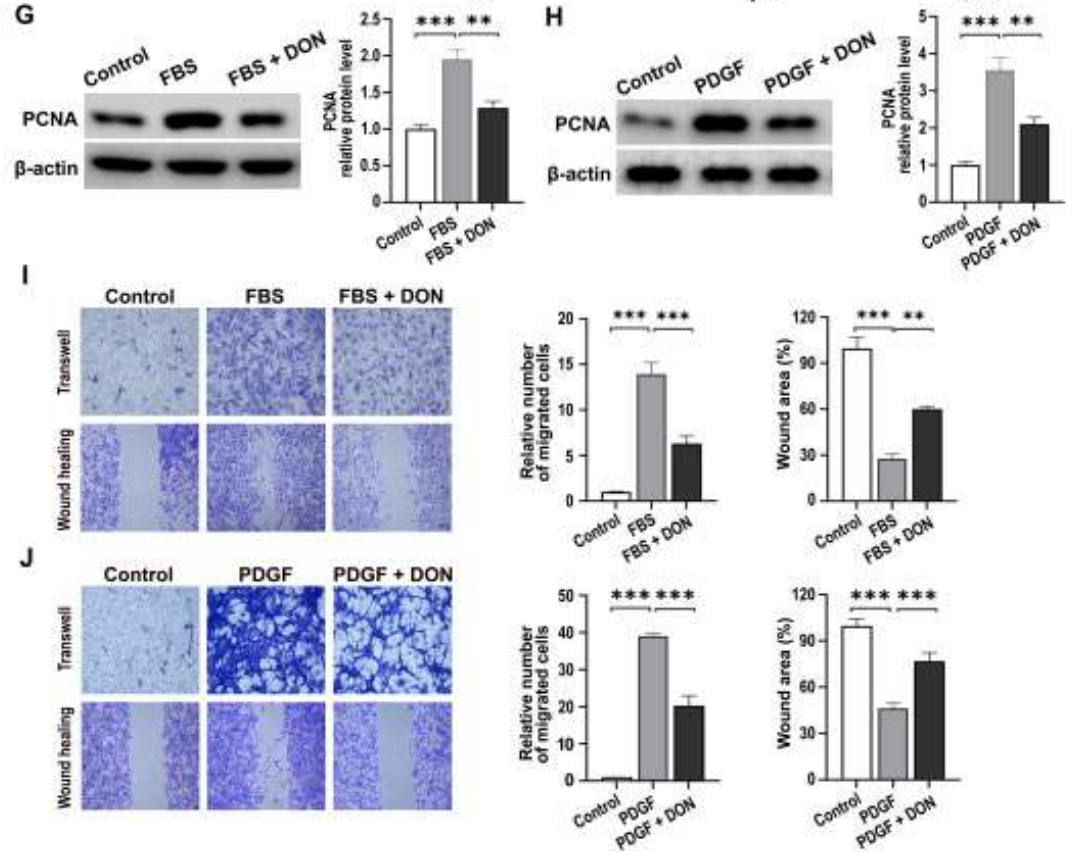

Figure 4. DON delays growth factor-induced cell cycle progression and inhibits proliferation and migration of VSMCs. (A,B) Representative western blots showing the effects of DON on the level of cyclin D1 in VSMCs stimulated with FBS (A) and PDGF (B). Data in bar graphs are mean \pm SEM of three independent measurements. (C,D) Histogram showing the cell cycle distribution of VSMCs stimulated with FBS (C) and PDGF (D). (E,F) Effects of DON on proliferation of VSMCs stimulated with FBS (E) and PDGF (F). (G,H) Representative western blots showing the effects of DON on the level of PCNA in VSMCs stimulated with FBS (G) and PDGF (H). Data in bar graphs are mean \pm SEM of three independent measurements. (I,J) Transwell migration (upper) and wound healing assays (lower) showing the effects of DON on migration of VSMCs stimulated with FBS (I) and PDGF (J). Data in bar graphs are mean \pm SEM of three independent measurements. ${ }^{*} p<0.05,{ }^{* *} p<0.01$, and ${ }^{* * *} p<0.001$.

Given that DON inhibited FBS- and PDGF-stimulated mitochondrial respiration, mTORC1 activity, and glycolysis, we next investigated its effects on proliferation and migration of VSMCs. As expected, DON significantly reduced FBS- and PDGF-stimulated proliferation of VSMCs and the level of proliferating cell nuclear antigen (PCNA) (Figure 4E-H). 
Wound healing and Transwell chamber assays showed that DON markedly attenuated FBS- and PDGF-stimulated migration of VSMCs (Figure 4I,J).

\subsection{JHU-083 Attenuates Carotid Artery Ligation-Induced Neointimal Hyperplasia}

Finally, we investigated whether JHU-083, a DON-derived prodrug, affects proliferation and migration of VSMCs after carotid artery ligation in mice. Representative cross-sections of arteries showed neointima formation and substantial narrowing of the arterial lumen after carotid artery ligation for 4 weeks. The intimal area was significantly smaller and the ratio of the neointimal layer to the medial layer was significantly lower in the JHU-083-treated group than in the ligation only group (Figure 5A,B). Consistent with the effects of DON in vitro, JHU-083 decreased p-4E-BP(T37/46), HIF- $1 \alpha$, and PCNA staining in arteries after carotid artery ligation (Figure 5C,D).

A

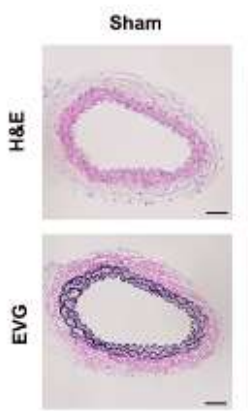

C
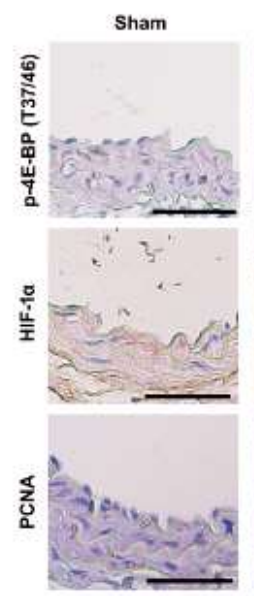

D

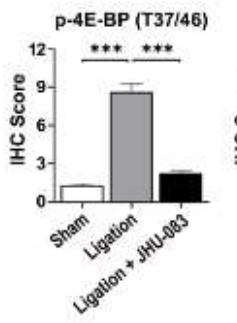

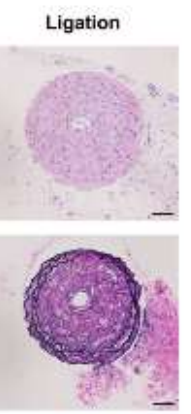
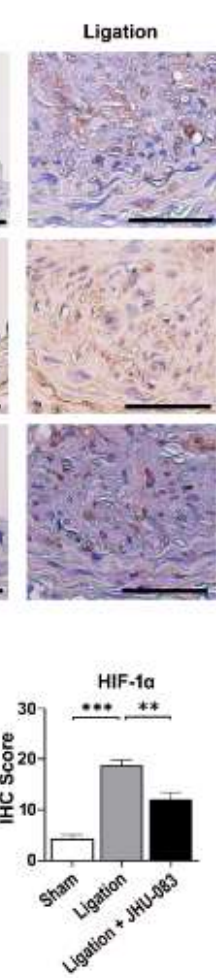
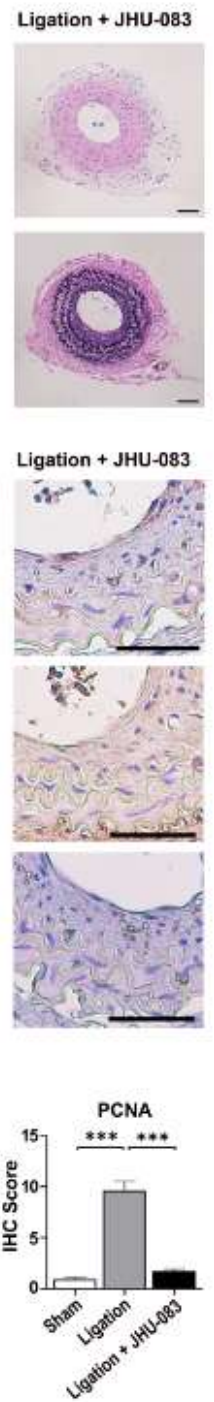

B
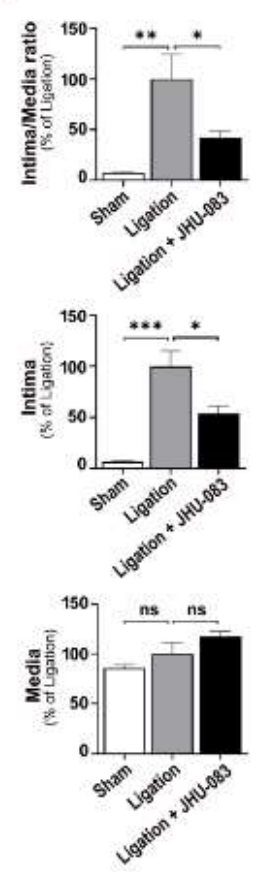

Figure 5. JHU-083 attenuates carotid artery ligation-induced neointimal hyperplasia. (A) Representative cross-sections of mouse carotid arteries stained with H\&E and Elastic-van Gieson (EVG), Scale bar represents $50 \mu \mathrm{m}$. (B) The relative ratios of the neointimal and medial areas, the intimal area, and the medial area quantified by morphometric analysis. Data are expressed as the mean \pm SEM ( $n=4-5$ per group). (C) Immunohistochemical staining of PCNA, p-4E-BP(Thr37/46) and HIF-1 $\alpha$ in mouse carotid arteries. (D) Cells immunohistochemically positive for PCNA, p-4E-BP(Thr37/46), and HIF- $1 \alpha$ in arteries were quantified. Data are expressed as mean \pm SEM ( $n=4-5$ per group). Scale bar represents $50 \mu \mathrm{m}$. NS, not significant; ${ }^{*} p<0.05,{ }^{* *} p<0.01$, and ${ }^{* * *} p<0.001$. 


\section{Discussion}

Our study revealed that the glutamine antagonist DON markedly inhibits growth factor-stimulated proliferation and migration of VSMCs. We demonstrated that the efficacy of DON was attributable to simultaneous suppression of FBS- or PDGF-stimulated glycolysis and oxidative phosphorylation as well as downregulation of mTORC1 activity in VSMCs. Finally, we showed that administration of JHU-083 attenuated neointimal hyperplasia after carotid artery ligation in mice.

mTORC1 is a master cell growth factor, and an inhibitor of this protein and rapamycin are commonly used in clinical settings to control VSMCs overgrowth-induced restenosis $[28,30]$. Glutaminolysis has a dual role in cancer cells; it sustains the TCA cycle and activates mTORC1 [26]. Inhibition of glutaminolysis by DON prevents GTP loading of RagB and lysosomal translocation and subsequent activation of mTORC1 [26]. In contrast to the inhibitory effect of DON on glutaminolysis, a more recent study reported that JHU083 reduces glioma cell proliferation by disrupting mTORC1 signaling, but the effect is independent of glutaminolysis [31]. Consistent with these previous results showing the inhibitory effects of a glutamine antagonist on mTORC1 activity, we observed that DON abrogated growth factor-induced mTORC1 activity in VSMCs. mTORC1-induced signaling pathways also regulate cyclin D1 protein expression and cell cycle progression from G to $S$ phase independent of gene transcription [29,32]. Based on our finding that DON decreased cyclin D1 expression and induced cell cycle arrest, the reduction of growth factorstimulated VSMC growth is attributable to disruption of mTORC1 by DON. Although the glutamine antagonist JHU-083 prevented ligation-induced occlusion, it did not completely restore the overall organization of the tissue induced by ligation. Considering that a previous report showed that DON and rapamycin had an additive effect on lymphocyte proliferation, with subsequent suppression of arthritis in SKG mice [33] it is expected that JHU-083 and rapamycin may additively affect the inhibition of VSMC proliferation and migration in vitro and in vivo. Furthermore, given that the mTOR pathway is implicated in stress fiber formation and focal adhesion formation [34,35], it might be worthwhile to investigate whether DON affects FBS- and PDGF-induced changes in stress fibers and focal adhesion formation in VSMCs.

Increased glycolysis appears to be critical for the bioenergetic shift that occurs during proliferation and migration of VSMCs $[8,10]$. Growth factor-treated VSMCs exhibit enhanced glycolytic flux and express markers of the synthetic VSMC phenotype [5,36,37]. We previously identified LDHA, which catalyzes the final step of glycolysis, as a therapeutic target for uncontrolled proliferation and migration of VSMCs [38]. Considering that mTORC1 reinforces the glycolytic program by increasing translation of HIF- $1 \alpha$ mRNA and stabilizing HIF- $1 \alpha$ expression [39], we assume that inhibition of mTORC1 by DON suppresses glycolysis in VSMCs in response to FBS or PDGF. We showed that upregulation of HIF- $1 \alpha$ and glycolysis in response to growth factor stimulation was attenuated by glutamine blockade. Together, our results demonstrate that glutamine blockade inhibits not only mTORC1 activity but also HIF- $1 \alpha$ expression, which in turn downregulates transcription of HK2 and LDHA $[7,36]$. Considering that proliferation of VSMCs and neointima formation are dependent on activation of glutamine uptake [40], simultaneous reduction of glutaminolysis and glycolysis may be a promising strategy to ameliorate constriction of vessel lumens during atherosclerosis and restenosis.

In conclusion, the present study shows that the glutamine antagonist DON inhibits proliferation and migration of growth factor-stimulated VSMCs by simultaneously suppressing FBS- and PDGF-stimulated glycolysis and oxidative phosphorylation. This study suggests that glutamine antagonists can be used to treat vascular occlusive disease.

\section{Materials and Methods}

\subsection{Murine Model of Carotid Artery Ligation}

Carotid artery ligation-induced neointimal hyperplasia was established in male C57BL/6J mice, as previously described [41]. Briefly, the unilateral common carotid 
artery was surgically ligated with a 5.0 suture tied near the distal bifurcation. JHU-083 was dissolved in sterile phosphate-buffered saline (PBS) immediately before treatment for in vivo studies [42]. Mice were administered JHU-083 via oral gavage $(0.6 \mathrm{mg} / \mathrm{kg} /$ day, 6 days per week for 4 weeks). Arteries proximal to the ligation site were analyzed by hematoxylin and eosin (H\&E) staining and an Elastic-van Gieson (EVG) staining (Abcam, Cambridge, UK). The cross-sectional intimal and medial areas were quantified using ImageJ software (National Institutes of Health, Bethesda, MD, USA). The intima-to-media ratio was calculated from the mean of these measurements.

\subsection{Immunohistochemistry}

Immunohistochemistry (IHC) was performed on formalin-fixed, paraffin-embedded tissue sections, as previously described [38]. The sections were incubated with anti-PCNA, (1:1000, Cell Signaling Technology, Beverly, MA, USA) anti-p-4E-BP1 (Thr37/46) (1:1000, Cell Signaling Technology) and anti-HIF-1 $\alpha$ (1:500, R\&D Systems, Minneapolis, MN, USA). The sections were visualized with diaminobenzidine (Liquid DAB+ Substrate Chromogen System; Dako, Carpinteria, CA, USA) and counterstained with Mayer's hematoxylin (Merck Inc., Darmstadt, Germany) at room temperature for $40 \mathrm{~s}$. Images of each group were obtained using a light microscope with CellSens Entry 1.9 imaging system (Olympus Corporation, Tokyo, Japan). Immunopositive cells and/or areas are shown in brown.

\subsection{Cell Culture}

VSMCs were isolated from the aortas of male Sprague-Dawley rats (weight, 90-100 g) according to a previously published method [43]. Briefly, the aortic tissues were carefully harvested and cut into pieces. The tissue pieces were immersed in low-glucose Dulbecco's modified Eagle's medium (DMEM; Hyclone, South Logan, UT, USA) containing 20\% FBS (Hyclone) and $1 \%$ penicillin/streptomycin at $37^{\circ} \mathrm{C}$ with $5 \% \mathrm{CO}_{2}$. Non-adherent cells were removed by replacing the medium every other day. All VSMCs used in this study were between the fourth and ninth passages.

\subsection{Cell Counting}

Primary VSMCs were cultured for $18 \mathrm{~h}$ under serum-starved conditions and then incubated for $24 \mathrm{~h}$ in the presence or absence of $10 \%$ FBS or PDGF-BB $(20 \mathrm{ng} / \mathrm{mL})$ with $5 \mu \mathrm{M}$ DON (Selleckchem, Houston, TX, USA). Cells were harvested and the total cell numbers were determined by the trypan blue exclusion assay using a hemocytometer under a microscope.

\subsection{Western Blot Analysis}

Western blot analysis was performed as previously described [44]. Proteins in cell lysates were separated by SDS-PAGE and transferred to PVDF membranes (Millipore, MA, USA). The membranes were incubated with primary antibodies specific for the following proteins: p-p70S6K (Thr389), p70S6K, p-4E-BP1 (Ser65), p-4E-BP1 (Thr37/46), 4E-BP1, PCNA, cyclin D1, LDHA, and HK2 (Cell Signaling Technology, Beverly, MA, USA); HIF-1 $\alpha$ (R\&D Systems, Minneapolis, MN, USA); and $\beta$-actin (Sigma, Saint Louis, MO, USA). After three washes in TBST, membranes were incubated with horseradish peroxidase-conjugated secondary antibodies (Santa Cruz Biotechnology, Dallas, TX, USA). Immunoreactive bands were detected with enhanced chemiluminescence reagent (BioNote, Gyeonggi-do, Korea) and visualized using an Amersham Imager 600 (GE Healthcare, Chicago, IL, USA). Band intensity was quantified using Image J software (National Institutes of Health, Bethesda, MD, USA).

\subsection{Migration Assays}

For the wound healing assay, VSMCs $\left(1 \times 10^{5}\right.$ cells) were plated onto 6-well plates and serum-starved for $18 \mathrm{~h}$. An artificial wound (scratch) was generated using a $200 \mu \mathrm{L}$ pipette tip. The cells were incubated with or without $5 \mu \mathrm{M}$ DON for $24 \mathrm{~h}$ in the presence or 
absence of $10 \%$ FBS or PDGF-BB $(20 \mathrm{ng} / \mathrm{mL})$. When the wound had closed, cells were fixed in $4 \%$ paraformaldehyde and stained with $0.05 \%$ crystal violet. For the Transwell migration assay, VSMCs $\left(1 \times 10^{4}\right.$ cells) were seeded onto the microporous membrane $(8.0 \mu \mathrm{m})$ in the upper chamber of the Transwell ${ }^{\circledR}$ (Corning Incorporated, New York, NY, USA). Cells were serum-starved for $18 \mathrm{~h}$ and then incubated with or without $5 \mu \mathrm{M} \mathrm{DON}$ for $24 \mathrm{~h}$ in the presence or absence of $20 \%$ FBS or PDGF-BB $(20 \mathrm{ng} / \mathrm{mL})$. Cells that had not migrated in the upper chamber were gently removed using a cotton swab. Cells that had migrated through the membrane to the lower chamber were fixed in methanol and stained with $0.05 \%$ crystal violet. Quantification of the transwell migration and wound area were performed using Image J software (National Institutes of Health, Bethesda, MD, USA).

\subsection{Flow Cytometric Analysis}

Cells were synchronized in G1 phase by serum starvation for $6 \mathrm{~h}$, and then incubated with or without $5 \mu \mathrm{M}$ DON for $12 \mathrm{~h}$ in the presence or absence of $10 \%$ FBS or PDGF-BB ( $20 \mathrm{ng} / \mathrm{mL}$ ). Thereafter, cells were trypsinized, washed with cold PBS containing $2 \%$ FBS, fixed for $1 \mathrm{~h}$ in $70 \%$ cold $\left(-20^{\circ} \mathrm{C}\right)$ ethanol, and stained for $30 \mathrm{~min}$ with PI/RNase Staining Buffer (BD Pharmingen, NJ, USA) in the dark. The percentage of cells in each phase of the cell cycle (G1, S, and G2/M) was analyzed using an Accuri ${ }^{\mathrm{TM}}$ C6 Plus flow cytometer (BD Bioscience, San Jose, CA, USA). Data were analyzed using FlowJo V10 software (Tree Star, Inc., Ashland, OR, USA).

\subsection{Measurement of the OCR and ECAR}

The bioenergetic properties of primary VSMCs under different conditions were determined using a XF-24 Seahorse extracellular flux analyzer (Seahorse Bioscience, North Billerica, MA, USA). Cells were seeded into a Seahorse XF24 plate, starved for $18 \mathrm{~h}$, and then incubated for $24 \mathrm{~h}$ with or without $5 \mu \mathrm{M}$ DON in the presence or absence of $10 \%$ FBS or PDGF-BB $(20 \mathrm{ng} / \mathrm{mL})$. For the Mito Stress assay, the culture medium was replaced by Seahorse XF DMEM supplemented with $5.55 \mathrm{mM}$ glucose and $1 \mathrm{mM}$ sodium pyruvate, and cultures were pre-incubated for $1 \mathrm{~h}$ at $37^{\circ} \mathrm{C}$ in a $\mathrm{CO}_{2}$-free incubator before measurements. Changes in cellular respiration were assessed over time upon consecutive injections of $1 \mu \mathrm{M}$ oligomycin (Sigma, Saint Louis, MO, USA), $2 \mu \mathrm{M}$ carbonyl cyanide 3-chlorophenylhydrazone (CCCP; Sigma, Saint Louis, MO, USA), and $1 \mu \mathrm{M}$ rotenone (Sigma, Saint Louis, MO, USA) at the indicated timepoints. For the Glycolysis Stress assay, the culture medium was replaced by Seahorse XF DMEM containing $5.55 \mathrm{mM}$ glucose before measurements. Cultures were equilibrated for $1 \mathrm{~h}$ at $37^{\circ} \mathrm{C}$ in a $\mathrm{CO}_{2}$-free incubator immediately before the XF assay. $\mathrm{pH}$ changes of the medium were determined in real-time upon sequential treatment with $10 \mathrm{mM}$ glucose (Sigma), $1 \mu \mathrm{M}$ oligomycin (Sigma, Saint Louis, MO, USA), and $100 \mathrm{mM}$ 2-deoxyglucose (2-DG; Sigma, Saint Louis, MO, USA). The OCR and ECAR were calculated automatically using the program of the Seahorse XF Mito Stress Test and Seahorse XF Glycolysis Stress Test according to the manufacturer's protocol, respectively.

\subsection{Statistical Analysis}

All values in the graphs represent the mean \pm standard error of the mean (SEM). A one-way analysis of variance followed by Dunnett's multiple comparison test was used to assess differences between groups (GraphPad Prism 8.0, San Diego, CA, USA).

Author Contributions: H.Y.P. performed the experiments and prepared original draft. M.-J.K. performed the experiments. S.L. (Seunghyeong Lee), J.J., S.L. (Sungwoo Lee) and J.-G.K. executed and interpreted the data. Y.-K.C. and K.-G.P. analyzed data, wrote, reviewed and revised the manuscript. All authors have read and agreed to the published version of the manuscript. 
Funding: This work was supported by the National Research Foundation of Korea (NRF) grant NRF-2020R1A5A2017323, funded by the Ministry of Science and ICT; grant HI15C0001 from the Korea Health Technology R\&D Project through the Korea Health Industry Development Institute, funded by the Ministry of Health and Welfare; and the Kyungpook National University Development Project Research Fund, 2018.

Institutional Review Board Statement: All animal procedures were approved on 4th of November 2020 by the Institutional Animal Care and Use Committee (IACUC) of Kyungpook National University (KNU2020-0124-2).

Informed Consent Statement: Not applicable.

Data Availability Statement: Not applicable.

Conflicts of Interest: The authors declare no conflict of interest. The funders had no role in the design of the study; in the collection, analyses, or interpretation of data; in the writing of the manuscript, or in the decision to publish the results.

\section{References}

1. Brozovich, F.; Nicholson, C.; Degen, C.; Gao, Y.Z.; Aggarwal, M.; Morgan, K. Mechanisms of Vascular Smooth Muscle Contraction and the Basis for Pharmacologic Treatment of Smooth Muscle Disorders. Pharmacol. Rev. 2016, 68, 476-532. [CrossRef] [PubMed]

2. Davis-Dusenbery, B.N.; Wu, C.; Hata, A. Micromanaging Vascular Smooth Muscle Cell Differentiation and Phenotypic Modulation. Arter. Thromb. Vasc. Biol. 2011, 31, 2370-2377. [CrossRef] [PubMed]

3. Chang, S.; Song, S.; Lee, J.; Yoon, J.; Park, J.; Choi, S.; Park, J.-K.; Choi, K.; Choi, C. Phenotypic Modulation of Primary Vascular Smooth Muscle Cells by Short-Term Culture on Micropatterned Substrate. PLoS ONE 2014, 9, e88089. [CrossRef] [PubMed]

4. Huang, J.; Parmacek, M.S. Modulation of smooth muscle cell phenotype: The other side of the story. Circ. Res. 2012, 111, 659-661. [CrossRef]

5. Shi, J.; Yang, Y.; Cheng, A.; Xu, G.; He, F. Metabolism of vascular smooth muscle cells in vascular diseases. Am. J. Physiol. Circ. Physiol. 2020, 319, H613-H631. [CrossRef]

6. Werle, M.; Kreuzer, J.; Höfele, J.; Elsässer, A.; Ackermann, C.; Katus, H.A.; Vogt, A.M. Metabolic control analysis of the Warburg-effect in proliferating vascular smooth muscle cells. J. Biomed. Sci. 2005, 12, 827-834. [CrossRef]

7. Lambert, C.M.; Roy, M.; Robitaille, G.A.; Richard, D.E.; Bonnet, S. HIF-1 inhibition decreases systemic vascular remodelling diseases by promoting apoptosis through a hexokinase 2-dependent mechanism. Cardiovasc. Res. 2010, 88, 196-204. [CrossRef] [PubMed]

8. Heiss, E.H.; Schachner, D.; Donati, M.; Grojer, C.S.; Dirsch, V.M. Increased aerobic glycolysis is important for the motility of activated VSMC and inhibited by indirubin-3'-monoxime. Vasc. Pharmacol. 2016, 83, 47-56. [CrossRef] [PubMed]

9. Renard, C.B.; Bornfeldt, K.E. Human arterial smooth muscle cells rapidly deplete cell culture media of glucose. Diabetologia 2001, 44, 1067-1068.

10. Hall, J.L.; Chatham, J.C.; Eldar-Finkelman, H.; Gibbons, G.H. Upregulation of Glucose Metabolism during Intimal Lesion Formation Is Coupled to the Inhibition of Vascular Smooth Muscle Cell Apoptosis: Role of GSK3. Diabetes 2001, 50, 1171-1179. [CrossRef]

11. Schultz, K.; Fanburg, B.L.; Beasley, D. Hypoxia and hypoxia-inducible factor-1 $\alpha$ promote growth factor-induced proliferation of human vascular smooth muscle cells. Am. J. Physiol. Circ. Physiol. 2006, 290, H2528-H2534. [CrossRef] [PubMed]

12. Zhao, X.; Tan, F.; Cao, X.; Cao, Z.; Li, B.; Shen, Z.; Tian, Y. PKM2-dependent glycolysis promotes the proliferation and migration of vascular smooth muscle cells during atherosclerosis. Acta Biochim. Biophys. Sin. 2019, 52, 9-17. [CrossRef] [PubMed]

13. Mazat, J.-P.; Ransac, S. The Fate of Glutamine in Human Metabolism. The Interplay with Glucose in Proliferating Cells. Metab. 2019, 9, 81. [CrossRef] [PubMed]

14. Cantor, J.R.; Sabatini, D.M. Cancer Cell Metabolism: One Hallmark, Many Faces. Cancer Discov. 2012, 2, 881-898. [CrossRef]

15. Eelen, G.; De Zeeuw, P.; Simons, M.; Carmeliet, P. Endothelial Cell Metabolism in Normal and Diseased Vasculature. Circ. Res. 2015, 116, 1231-1244. [CrossRef]

16. Bertero, T.; Oldham, W.M.; Cottrill, K.A.; Pisano, S.; Vanderpool, R.R.; Yu, Q.; Zhao, J.; Tai, Y.; Tang, Y.; Zhang, Y.-Y.; et al. Vascular stiffness mechanoactivates YAP/TAZ-dependent glutaminolysis to drive pulmonary hypertension. J. Clin. Investig. 2016, 126, 3313-3335. [CrossRef]

17. Pettit, G.R.; Nelson, P.S. Synthesis of the Streptomyces ambofaciens antineoplastic constituent 6-diazo-5-oxo-L-norleucine. J. Org. Chem. 1983, 48, 741-744. [CrossRef]

18. Lemberg, K.M.; Vornov, J.J.; Rais, R.; Slusher, B.S. We're Not “DON” Yet: Optimal Dosing and Prodrug Delivery of 6-Diazo-5-oxoL-norleucine. Mol. Cancer Ther. 2018, 17, 1824-1832. [CrossRef]

19. Crosby, H.A.; Ihnat, M.; Miller, K.E. Evaluating the Toxicity of the Analgesic Glutaminase Inhibitor 6-Diazo-5-oxo-L-norleucine in vitro and on rat dermal skin fibroblasts. MOJ Toxicol. 2015, 1, 00005. [CrossRef] 
20. Rais, R.; Jančařík, A.; Tenora, L.; Nedelcovych, M.; Alt, J.; Englert, J.; Rojas, C.; Le, A.; Elgogary, A.; Tan, J.; et al. Discovery of 6-Diazo-5-oxo-l-norleucine (DON) Prodrugs with Enhanced CSF Delivery in Monkeys: A Potential Treatment for Glioblastoma. J. Med. Chem. 2016, 59, 8621-8633. [CrossRef]

21. Zhu, X.; Nedelcovych, M.T.; Thomas, A.G.; Hasegawa, Y.; Moreno-Megui, A.; Coomer, W.; Vohra, V.; Saito, A.; Perez, G.; Wu, Y.; et al. JHU-083 selectively blocks glutaminase activity in brain CD11b(+) cells and prevents depression-associated behaviors induced by chronic social defeat stress. Neuropsychopharmacology 2019, 44, 683-694. [CrossRef]

22. Ueki, N.; Lee, S.; Sampson, N.S.; Hayman, M.J. Selective cancer targeting with prodrugs activated by histone deacetylases and a tumour-associated protease. Nat. Commun. 2013, 4, 2735. [CrossRef] [PubMed]

23. Leone, R.D.; Zhao, L.; Englert, J.M.; Sun, I.-M.; Oh, M.-H.; Arwood, M.L.; Bettencourt, I.A.; Patel, C.H.; Wen, J.; Tam, A.; et al. Glutamine blockade induces divergent metabolic programs to overcome tumor immune evasion. Science 2019, 366, 1013-1021. [CrossRef] [PubMed]

24. Ren, L.; Ruiz-Rodado, V.; Dowdy, T.; Huang, S.; Issaq, S.H.; Beck, J.; Wang, H.; Hoang, C.T.; Lita, A.; Larion, M.; et al. Glutaminase-1 (GLS1) inhibition limits metastatic progression in osteosarcoma. Cancer Metab. 2020, 8, 4-13. [CrossRef]

25. Wu, F.; Lukinius, A.; Bergström, M.; Eriksson, B.; Watanabe, Y.; Långström, B. A mechanism behind the antitumour effect of 6-diazo-5-oxo-1-norleucine (DON): Disruption of mitochondria. Eur. J. Cancer 1999, 35, 1155-1161. [CrossRef]

26. Durán, R.V.; Oppliger, W.; Robitaille, A.M.; Heiserich, L.; Skendaj, R.; Gottlieb, E.; Hall, M.N. Glutaminolysis activates RagmTORC1 signaling. Mol. Cell 2012, 47, 349-358. [CrossRef]

27. Yecies, J.L.; Manning, B.D. Transcriptional Control of Cellular Metabolism by mTOR Signaling. Cancer Res. 2011, 71, 2815-2820. [CrossRef]

28. Marx, S.O.; Jayaraman, T.; Go, L.O.; Marks, A.R. Rapamycin-FKBP Inhibits Cell Cycle Regulators of Proliferation in Vascular Smooth Muscle Cells. Circ. Res. 1995, 76, 412-417. [CrossRef]

29. Cuyàs, E.; Corominas-Faja, B.; Joven, J.; Menendez, J.A. Cell Cycle Regulation by the Nutrient-Sensing Mammalian Target of Rapamycin (mTOR) Pathway. Methods in Molecular Biology. 2014, 1170, 113-144.

30. Martin, K.A.; Rzucidlo, E.M.; Merenick, B.L.; Fingar, D.C.; Brown, D.J.; Wagner, R.J.; Powell, R.J. The mTOR/p70 S6K1 pathway regulates vascular smooth muscle cell differentiation. Am. J. Physiol. Physiol. 2004, 286, C507-C517. [CrossRef]

31. Yamashita, A.S.; Rosa, M.D.C.; Stumpo, V.; Rais, R.; Slusher, B.S.; Riggins, G.J. The glutamine antagonist prodrug JHU-083 slows malignant glioma growth and disrupts mTOR signaling. Neuro-Oncology Adv. 2021, 3, vdaa149. [CrossRef]

32. Hidayat, S.; Yoshino, K.; Tokunaga, C.; Hara, K.; Matsuo, M.; Yonezawa, K. Inhibition of amino acid-mTOR signaling by a leucine derivative induces G1 arrest in Jurkat cells. Biochem. Biophys. Res. Commun. 2003, 301, 417-423. [CrossRef]

33. Ueda, Y.; Saegusa, J.; Okano, T.; Sendo, S.; Yamada, H.; Nishimura, K.; Morinobu, A. Additive effects of inhibiting both mTOR and glutamine metabolism on the arthritis in SKG mice. Sci. Rep. 2019, 9, 6374. [CrossRef]

34. Lee, F.-Y.; Zhen, Y.-Y.; Yuen, C.-M.; Fan, R.; Chen, Y.-T.; Sheu, J.-J.; Chen, Y.-L.; Wang, C.-J.; Sun, C.-K.; Yip, H.-K. The mTOR-FAK mechanotransduction signaling axis for focal adhesion maturation and cell proliferation. Am. J. Transl. Res. $2017,9,1603-1617$.

35. Liu, L.; Chen, L.; Chung, J.; Huang, S. Rapamycin inhibits F-actin reorganization and phosphorylation of focal adhesion proteins. Oncogene 2008, 27, 4998-5010. [CrossRef]

36. Perez, J.; Hill, B.G.; Benavides, G.A.; Dranka, B.P.; Darley-Usmar, V.M. Role of cellular bioenergetics in smooth muscle cell proliferation induced by platelet-derived growth factor. Biochem. J. 2010, 428, 255-267. [CrossRef]

37. Dong, X.; Wu, D.; Zhang, Y.; Jia, L.; Pan, X.; Sun, J.; Pan, L.-L. Cathelicidin Modulates Vascular Smooth Muscle Cell Phenotypic Switching through ROS/IL-6 Pathway. Antioxidants 2020, 9, 491. [CrossRef]

38. Kim, J.-H.; Bae, K.-H.; Byun, J.-K.; Lee, S.; Kim, J.-G.; Lee, I.K.; Jung, G.-S.; Lee, Y.M.; Park, K.-G. Lactate dehydrogenase-A is indispensable for vascular smooth muscle cell proliferation and migration. Biochem. Biophys. Res. Commun. 2017, 492, 41-47. [CrossRef]

39. Hudson, C.C.; Liu, M.; Chiang, G.G.; Otterness, D.M.; Loomis, D.C.; Kaper, F.; Giaccia, A.J.; Abraham, R.T. Regulation of Hypoxia-Inducible Factor $1 \alpha$ Expression and Function by the Mammalian Target of Rapamycin. Mol. Cell. Biol. 2002, 22, 7004-7014. [CrossRef]

40. Osman, I.; He, X.; Liu, J.; Dong, K.; Wen, T.; Zhang, F.; Yu, L.; Hu, G.; Xin, H.-B.; Zhang, W.; et al. TEAD1 (TEA Domain Transcription Factor 1) Promotes Smooth Muscle Cell Proliferation Through Upregulating SLC1A5 (Solute Carrier Family 1 Member 5)-Mediated Glutamine Uptake. Circ. Res. 2019, 124, 1309-1322. [CrossRef]

41. Kumar, A.; Lindner, V. Remodeling With Neointima Formation in the Mouse Carotid Artery after Cessation of Blood Flow. Arter. Thromb. Vasc. Biol. 1997, 17, 2238-2244. [CrossRef]

42. Hanaford, A.R.; Alt, J.; Rais, R.; Wang, S.Z.; Kaur, H.; Thorek, D.L.; Eberhart, C.G.; Slusher, B.S.; Martin, A.M.; Raabe, E.H. Orally bioavailable glutamine antagonist prodrug JHU-083 penetrates mouse brain and suppresses the growth of MYC-driven medulloblastoma. Transl. Oncol. 2019, 12, 1314-1322. [CrossRef]

43. Chamley-Campbell, J.; Campbell, G.R.; Ross, R. The smooth muscle cell in culture. Physiol. Rev. 1979, 59, 1-61. [CrossRef]

44. Lee, S.; Byun, J.; Park, M.; Kim, S.W.; Lee, S.; Kim, J.; Lee, I.; Choi, Y.; Park, K. Melatonin inhibits vascular smooth muscle cell proliferation and apoptosis through upregulation of Sestrin2. Exp. Ther. Med. 2020, 19, 3454-3460. [CrossRef] 